\title{
Los Insectos de Galápagos
}

\author{
Olivier Dangles \\ Escuela de Ciencias Biológicas de la Pontificia Universidad Católica del Ecuador. \\ odangles@puce.edu.ec
}

Los estudios de entomología en las Galápagos se iniciaron con el viaje de Charles Darwin en 1835. Cuando él llegó al Archipiélago de Colón era la época seca, por tanto la abundancia de insectos era relativamente baja. Eso explica posiblemente por qué el investigador inglés no se interesó tanto en los insectos y no realizó significativos comentarios de ellos en su obra, en comparación con otros grupos. Después de Darwin, más de 20 expediciones entomológicas fueron realizadas en las Galápagos; una de ellas fue de la Academia de California en 1905-1906 que estudió las comunidades de insectos durante un año entero. Todos estos trabajos fueron recopilados en un libro por Peck (2001), que es una buena referencia para las personas interesadas en esta temática.

Hoy en día, se conoce que en las Islas Galápagos existen más de 2000 especies de insectos dentro de alrededor de 1000 géneros, y se estima que $90-95 \%$ de las especies ya fueron colectadas. El $40 \%$ de estas especies y el $2.8 \%$ de estos géneros son endémicos (Peck, 2001). La diversidad de insectos en las Galápagos es relativamente baja en relación con el tamaño del Archipiélago, lo que se debe a varios factores como la escasez de agua dulce, el alto nivel de auto-polinización en las plantas y el clima bastante árido; mucho más en relación, que en otros Archipiélagos como Hawai o las Islas Canarias donde se tiene una entomofauna más diversa (por ejemplo, tienen 4 veces más de especies de Coleópteros). Al nivel del Archipiélago, esta diversidad esta elacionada con el tamaño y la altura de las islas pero no con su edad. Eso se debe a que las islas más grandes (las cuales son también las más altas por el crecimiento de la lava de los volcanes) tienen una mayor diversidad de hábitats y gradientes ambientales.

Aunque las Islas Galápagos fueron un laboratorio de la teoría de la evolución por muchas especies, el asunto es más complicado en lo que se refiere al área de insectos. $\mathrm{Si}$ se reportan casos de especiación de insectos en el Archipiélago (por ejemplo 17 spp de Coleópteros Tenebrionidae dentro del genero Stomion, Finston \& Peck 2004), sin embargo, el nivel de especiación de los insectos es relativamente bajo, estimado a $1.15 \mathrm{sp} /$ género y concierne solamente $5 \%$ de los géneros. Los estudios actuales de biogeografía de los insectos en las Galápagos son complicados por el efecto de los humanos. Como reporta el entomólogo R. Silberglied (1978): "Indudablemente la distribución de muchas especies [de insectos] ha cambiado debido a la dispersión 
inter-islas por barco y especies exóticas que han arribado a las islas y se han dispersado entre ellas por la misma vía ...". De hecho, un estudio reciente llevado a cabo por la Estación Científica Charles Darwin ha demostrado que un barco grande de turistas con su gran cantidad de luz externa podría atraer y transportar hasta 367542 insectos al año entre las islas, en mayor abundancia Lepidópteros pero también Himenópteras y Dípteras (RoqueAlbelo et al. 2006). Además, desde el siglo pasado, el número de especies de insectos exóticos reportado en las Galápagos ha seguido un crecimiento exponencial, de menos de 50 especies en los años 1920 a más de 300 en los años 2000 (Peck 2001).

Aunque relativamente poco estudiada en comparación de otros grupos, la entomofauna de las Galápagos posee mucho interés para los científicos, tanto del lado académico para mejorar nuestro conocimiento sobre la evolución de los insectos en las Islas, como del punto de vista aplicado para proteger la fauna y la flora endémicas del Archipiélago de Colón o Islas Galápagos.

\section{BIBLIOGRAFÍA}

Finston TL and Peck SB 2004. Speciation in Darwin's darklings: taxonomy and evolution of Stomion beetles in the Galápagos Islands, Ecuador (Insecta: Coleoptera: Tenebrionidae) Zoological Journal of the Linnean Society, 141, 135-152.

Peck S.B. 2001. Smaller orders of insects of the Galapagos Islands, Ecuador: Evolution, Ecology, and Diversity. National Research Council, Research Press, Canada, 278 pp.

Roque-Albelo L, Berg M, and Galarza M. 2006. "Polizontes peligrosos", Dispersión de insectos entre las Islas Galápagos en Barcos de Turismo. Informe de Investigación Fundación Charles Darwin para las Islas Galápagos A.I.S.B.L., 23pp.

Silberglied, R. 1978. Inter-Island transport of insect aboard ships in the Galapagos Islands. Biological Conservation, 13: 273-278. 